\title{
Patient partnership falling to wayside in COVID-19 research rush
}

\author{
n Cite as: CMAJ 2021 July 12;193:E1054-5. doi: 10.1503/cmaj.1095950
}

Posted on cmajnews.com on June 22, 2021

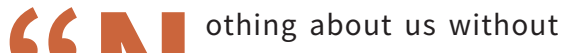 us" has become a common refrain over the past} decade as patients and communities have pushed for a greater say in health care research and decision-making. But now some fear pandemic expediencies are eroding hard-won advances in patient partnership.

COVID-19 had a "catastrophic" impact on patient engagement in health research, says Bertrand Lebouché, a clinicianresearcher at McGill University Health Centre.

Prior to the pandemic, funding bodies increasingly required investigators to involve patients throughout the research process - from setting priorities to translating results into practice. "It gives an opportunity for the patient to become part of the solution," says Lebouché.

With COVID-19, these requirements mostly fell by the wayside. "Of course, COVID-19 is an exceptional circumstance. But you can ask the population, 'What do you think? What do you need?' And this was not done."

Patient partnership can be costly, time consuming and complicated, so it's understandable why it stalled in the first months of the pandemic. Yet more than a year later, many funding calls for COVID-19 research still don't mention the need to include patients or the public as partners.

"We need 2 step up + not lose ground," tweeted Kerry Kuluski, a research chair in patient and family-centred care at the Institute for Better Health at Trillium Health Partners.

COVID-19 highlighted huge disparities in health by income, race, and other social determinants, noted Cynthia Weijs, a CIHR Health System Impact Fellow at

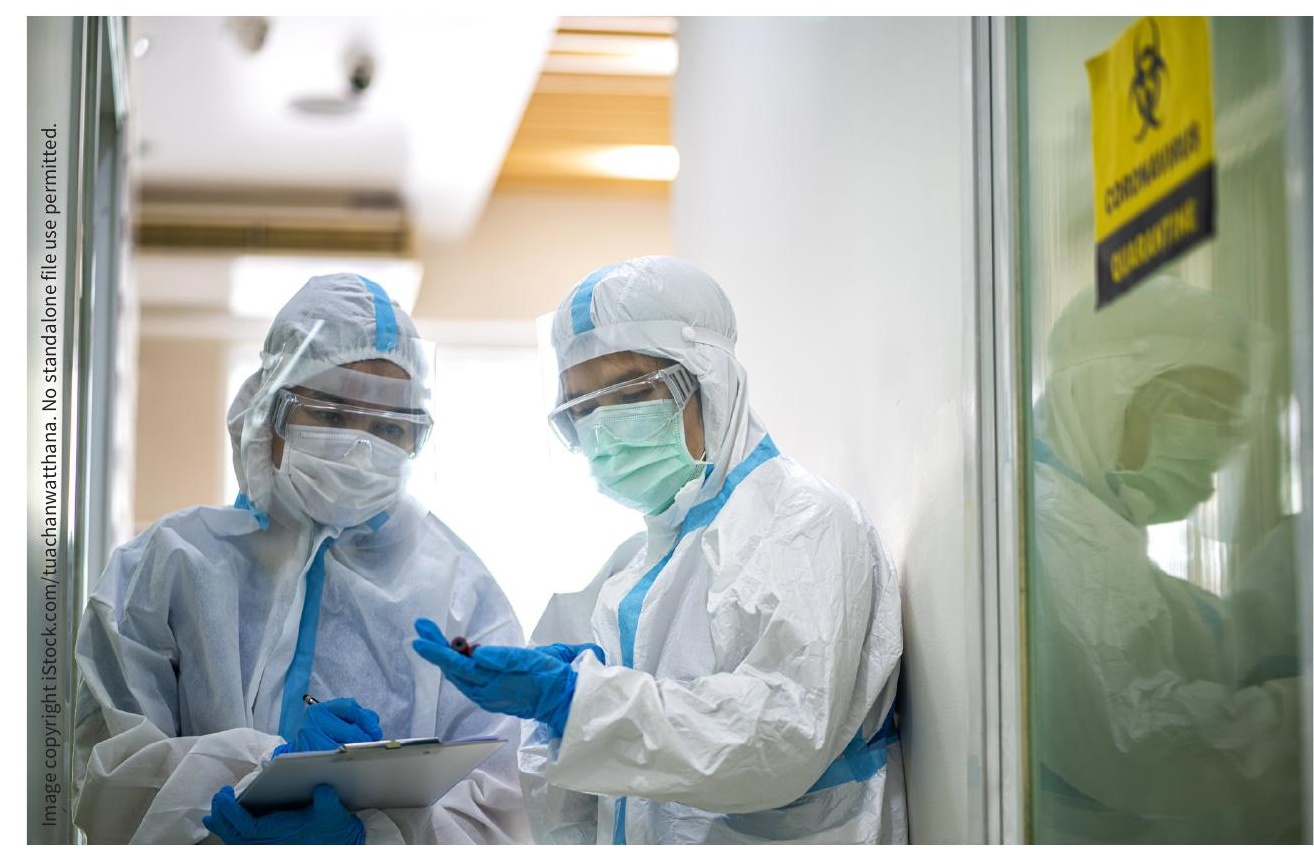

Health researchers and funders have pivoted quickly during the pandemic, but some say patients are being left out.

Alberta Health Services and the University of Calgary. "So, it was a bit of a surprise to see so many funding calls that fall back on the expertise of researchers," rather than centring the people hit hardest by the pandemic.

According to Weijs, the pandemic has also raised upstream questions about the purpose of patient partnership. "What is the goal of involving patients and the public in research? Are we trying to share power with them? Because right now, that's not how it works."

The Canadian Institutes of Health Research (CIHR) has been a major driver of patient partnership through its Strategy for Patient-Oriented Research (SPOR). However, the federal funding agency acknowledged that it had to simplify processes to respond to COVID-19 quickly.
"This meant that patient engagement was included as a component in some but not all of our COVID competitions," the agency told CMAJ.

According to $\mathrm{ClHR}$, one of the biggest challenges to involving patients was a lack of time to develop meaningful relationships and mobilize partnerships developed before the pandemic. However, the agency emphasized its ongoing commitment to supporting patient partnership.

Lebouché, who holds a SPOR chair in innovative clinical trials in HIV care, received an emergency grant from the McGill Interdisciplinary Initiative in Infection and Immunity to adapt a smartphone application for patients with COVID-19.

Involving patients in the project was difficult "because it was all through Zoom right from the start," Lebouché 
says. "I am also used to engaging patients with a chronic condition, where we have a long-term relationship." The acute nature of COVID-19 meant "we had to develop a new strategy for participation and engagement."

Researchers who were already partnering with patients before the pandemic could draw on those relationships to help them adapt their work "in a good and grounded way," says Isabel Jordan, a British Columbiabased patient partner and a founding member of the Rare Disease Foundation.

However, Jordan says she is concerned about investigators who are building partnerships from scratch. "The pandemic creates another barrier to doing it well. If you don't have connections into the patient community, it's harder to create that trust and that safety virtually."

For all the lip service paid to the value of patient perspectives, Jordan and others have noted a "shrinking back" from partnership during the pandemic. "Suddenly, we're not part of the team anymore. For a lot of people, it was a shock because there was a feeling like we had gotten to the right place."

Even so, patients with COVID-19 have played an instrumental role in expanding understanding of the disease. Early in the pandemic, informal patient support groups raised alarm about a host of long-term symptoms, now known as Long COVID, challenging clinicians' assumptions that most people recovered within two weeks. "Their findings have made a large impact... and highlight the critical importance of patient-led research," concluded Lisa McCorkell and coauthors in Pain Reports.

According to Lebouché, rebounding from the pandemic slump in patient partnership will require granting agencies to put out more funding competitions that specifically call for it. "Researchers follow the funding."
Without dedicated funding and training to support researchers in partnering with patients, Jordan says "from a financial point of view, it makes no sense to have patient partners."

"There are some people who are down on patient partnership, but I think often it doesn't work because people aren't given the tools to do it well," she says. "We get better answers to our health care questions when we include the perspectives of everybody who is using the system."

Abigail Cukier, Hamilton, Ont.

Content licence: This is an Open Access article distributed in accordance with the terms of the Creative Commons Attribution (CC BY-NC-ND 4.0) licence, which permits use, distribution and reproduction in any medium, provided that the original publication is properly cited, the use is noncommercial (i.e., research or educational use), and no modifications or adaptations are made. See: https://creativecommons.org/ licenses/by-nc-nd/4.0/ 